S. Durán, M. Duch, T. Patiño, A. Torres, O. Penon, R. Gómez-Martínez, L. Barrios, J. Esteve, C. Nogués, L. Pérez-García, J. A. Plaza,

"Technological development of intracellular Polysilicon-Chromium-Gold chips for orthogonal chemical functionalization",

Sensors and Actuators B, Vol. 209, pp. 212-224, 2015

https://doi.org/10.1016/j.snb.2014.11.077

https://www.sciencedirect.com/science/article/pii/S0925400514014609 


\title{
Technological development of intracellular Polysilicon-Chromium- Gold chips for orthogonal chemical functionalization
}

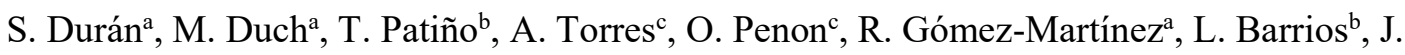 \\ Esteve $^{\mathrm{a}}$, C. Nogués ${ }^{\mathrm{b}}$, L. Pérez-García ${ }^{\mathrm{c}}$, J. A. Plaza ${ }^{\mathrm{a},{ }^{*}}$ \\ ${ }^{\text {a } I n s t i t u t o ~ d e ~ M i c r o e l e c t r o ́ n i c a ~ d e ~ B a r c e l o n a, ~ I M B-C N M ~(C S I C), ~ C a m p u s ~ U A B, ~ 08193, ~}$ \\ Cerdanyola, Barcelona, Spain. \\ ${ }^{\mathrm{b}}$ Department de Biologia Cel•lular, Fisiologia i Immunologia, Universitat Autònoma de \\ Barcelona, 08193, Bellaterra, Spain \\ ${ }^{\mathrm{c}}$ Department of Pharmacology and Therapeutical Chemistry and Institute of \\ Nanoscience and Nanotechnology UB (IN2UB), Universitat de Barcelona, Avda .Joan \\ XXIII s/n, 08028, Barcelona, Spain.
}

\begin{abstract}
Increasingly, advances in microtechnologies are focused on obtaining new chips intended for applications in fields such as nanomedicine and cell biology, taking advantage of the ability of microelectronics to manufacture devices with cell dimensions and a large variety of features. Here, we report a technology for the fabrication of multi-material chips, using polysilicon and gold as device layers, to be used as bi-functional cellinternalizable devices. In our case, one of the main technological challenges is to overcome the low adherence between these two materials, especially because of their small contact-area, only $9 \mu \mathrm{m}^{2}$. Thus, in order to circumvent this difficulty a chromium adherent-layer was deposited in between. After fabrication, the devices following this design can be successfully internalized inside living macrophages without affecting their viability. The advantage of having multiple material layers in one device is the potential to render multi-tasking chips, as once they are appropriately functionalized, we can provide the chip the ability of being bi-functional. Hence, and as a proof of concept, two different proteins, Wheat Germ Agglutinin (WGA) and Concanavalin (ConA), were
\end{abstract}


immobilized on the chip surface through self-assembled monolayers using orthogonal chemistry. The results of this work show a well-controlled fabrication, the bi-functional capabilities and no cell-toxicity of intracellular polysilicon-chromium-gold chips. These devices have a promising future as intracellular functional platforms for biosensing, drug delivery and diagnosis.

Keywords: Intracellular chips, silicon, chromium, gold, cell-internalization, orthogonal biofunctionalization

\section{Introduction}

Nowadays, semiconductor technologies are providing new tools, that extracellularly, can sense and control parameters in cell biology $[1,2]$. Relevant examples can be found from chemical to physical applications, for instance, biosensors [3], diagnosis devices [4], drug delivery systems [5] or even for cell mechanics [6].

By going further and taking advantage of the miniaturization capabilities of micro- and nanotechnologies, researchers are focusing their efforts on manufacturing devices small enough to be internalized inside single living cells $[7,8]$. However, to the date, the technological development for most valuable intracellular devices is currently under investigation by many researchers worldwide $[9,10]$. Following this line of research, we have previously demonstrated that microchips made of only one material, polysilicon, can be fabricated and internalized inside single living cells as intracellular barcodes [11] or as nanomechanical devices, [12] always showing a low cellular toxicity. Conversely, the chemical functionalization of these devices opens up new opportunities as intracellular actuators in cell biology. For instance, we reported that chemically functionalized silicon chips act as intracellular biomolecular sensors [13] or as 
extracellular tags which can be adhered to oocytes and embryos at different development stages [14-17].

Multifunctional nano- and micromaterials represent huge opportunities for developing new strategies to many scientific challenges, for instance, the advantages that offer in theranostics [18]. Nevertheless, multiple chemical biofunctionalization in a single device, allowing the fabrication of multipurpose tools, still remains a challenge. Achieving multifunctionality is possible mainly using self-assembly, a synthetic tool that allows the formation of ordered arrays at the nanoscale [19]. However, coadsorption of different compounds on surfaces often results on disordered mixed monolayers [20]. Spatially controlled functionalized surfaces are desirable, and, most of the times, patterning techniques rely mainly on lithographic methodologies: either microcontact printing, photolithography or nanografting, applied on monomaterial surfaces [21]. A different approach consists of performing material selective surface modification, using a combination of orthogonal chemistry [22] on polymaterial substrates. This methodology has been successful using nanoparticles $[23,24]$ and surfaces $[25,26]$, although it presents limitations for protein patterning and it has not been well developed on microfabricated particles [27].

In order to avoid the inconvenient of applying multi-functionalization procedures to a single-material chip, which could ruin the efficient control on the localization of the different biomolecules, chips composed of several materials were designed, that will then take advantage of orthogonal chemistry functionalization methodologies. Accordingly, microelectronic technologies are highly-suitable candidates for the fabrication of these multi-functional devices as they offer the possibility of depositing or growing many different materials as insulators, semiconductors and metals with high precision, well- 
known surface chemistry and many of them being biocompatible, generating a solid foundation for them to be used in cell biology applications.

In this paper we report the technology for the fabrication of chips made from a combination of polysilicon and gold layers through common semiconductor techniques, orthogonally functionalized and bio-compatible (Figure 1). Polysilicon and gold, common materials in MEMS industry, are selected as device materials because of their well-known chemistry [14,28-32] and biocompatibility [33]. In addition, gold has been used widely in chemical and biochemical sensors as a signal transducer based on its unique properties [34]. Nevertheless, one of the main issues in the applicability of gold thin films in microfabrication technology is their weak adhesion to silicon substrates [3537]. For this reason, we also consider the deposition of a chromium interlayer between the gold and polysilicon layers, due to its efficient capabilities as a material-adherent [3538]. Consequently, the intracellular biocompatibility of chromium as such device material should be also evaluated. Thus, a technological development for the fabrication of chips entirely made of chromium and their corresponding cell viability tests are also presented. Then, orthogonal chemistry protocols, using self-assembled monolayers of thiol and silane derivatives, for gold and silicon selective bi-functionalization of a suspension of polysilicon-chromium-gold chips are also developed. The main difference between single-functional platforms and the bi-functional platforms is that the bi-functionals could combine in situ two different biological activities, such as, biosensors, biomarkers or therapeutic agents. As a probe of concept, two different fluorescence labelled lectins have been immobilized due to their important role in carbohydrates recognition. Lastly, we investigate the capability of these devices of being cell-internalizable, as well as their biocompatibility inside living cells. 


\section{Material and methods}

\subsection{Fabrication of Polysilicon-Gold chips.}

Polysilicon and gold chips using semiconductor technologies were fabricated. The deposition of $1 \mu \mathrm{m}$-thick silicon oxide as a sacrificial layer on a $100 \mathrm{~mm} \varnothing$ p-type silicon wafer began the fabrication (Figure 2a and b). A $400 \mathrm{~nm}$ thick polysilicon layer was deposited (temperature $580^{\circ} \mathrm{C}$, pressure $350 \mathrm{mTorr}$ and a $\mathrm{SiH}_{4}$ flow rate of $40 \mathrm{sccm}$ ) on the wafer as first device-layer (Figure 2c). A photolithographic step created a photoresist inverse pattern (Figure 2d). Next, a $100 \mathrm{~nm}$-thick gold deposition layer was performed as the second device-layer (Figure 2e). A lift-off process was carried out for 7 minutes in an acetone solution, washing away the sacrificial photoresist. As explained below a long liftoff process was performed, as the bad adherence of gold over the polysilicon layer resulted in an unclear vision whether the main device layer (polysilicon) was exposed or longer times were needed. The target material, in this case gold, remained only in the regions where the material had a direct contact with the polysilicon substrate (Figure $2 \mathrm{f}$ ). A polysilicon dry etching for 25 seconds using the gold as a mask material finally defined the chips (Figure $2 \mathrm{~g}$ ). An array of $3 \mu \mathrm{m} \times 3 \mu \mathrm{m}$ chips separated $3 \mu \mathrm{m}$ was obtained. Lastly, the chips were released by a silicon oxide sacrificial etching in vapors of $49 \% \mathrm{HF}$ for 40 minutes, suspended in $96 \%$ ethanol, centrifuged at $14000 \mathrm{~g}$ for 5 minutes (MiniSpin Plus ${ }^{\circledR}$ ) and collected in eppendorfs for their posterior inspections (Figure $2 \mathrm{~h}$ ). 


\subsection{Fabrication of Chromium chips}

The fabrication process was initiated by the deposition of $1 \mu \mathrm{m}$-thick silicon oxide sacrificial layer on a $100 \mathrm{~mm} \varnothing$ p-type silicon wafer (Figure $3 \mathrm{a}$ and b). A photolithographic step defined an inverse photoresist pattern (Figure $3 \mathrm{c}$ and $\mathrm{d}$ ). Next, a $100 \mathrm{~nm}$-thick chromium deposition as a device layer was performed (Figure 3e). A liftoff process for 30 seconds was carried out to finally define the devices (Figure $3 \mathrm{f}$ ). The lift-off process for chromium chips was only 30 second-long, due to their perfectly deposited chromium layer and their thin chromium thickness that did not require longer times to easily arrive to the silicon oxide sacrificial layer. Finally, chips were released by a sacrificial etching of the silicon oxide layer in vapors of $49 \%$ HF for 40 minutes, suspended in $96 \%$ ethanol, centrifuged at 14000 g for 5 minutes (MiniSpin Plus ${ }^{\circledR}$ ) and collected in eppendorfs (Figure $3 \mathrm{~g}$ ).

\subsection{Fabrication of Polysilicon-Chromium-Gold chips}

Starting from $100 \mathrm{~mm} \varnothing$ p-type silicon wafer (Figure 4a), a $1 \mu \mathrm{m}$-thick silicon oxide sacrificial layer was deposited (Figure 4b). A 400 nm-thick polysilicon layer was performed (temperature $580^{\circ} \mathrm{C}$, pressure $350 \mathrm{mTorr}$ and a $\mathrm{SiH}_{4}$ flow rate of $40 \mathrm{sccm}$ ) as a first device layer (Figure 4c). Then, a photolithographic step created a photoresist inverse pattern (Figure 4d). Then $30 \mathrm{~nm}$-thick chromium layer (Figure 4e) was deposited as adherent interlayer and, finally, a $100 \mathrm{~nm}$-thick gold (Figure 4f) deposition defined the second device layer. Next, a lift-off process was carried out for 2 minutes in an acetone solution (Figure 4g). The lift-off process for the fabrication of polysilicon-chromium-gold was 2 minute-long. In this case, a longer time was used in contrast to that used for chromium chips due to their bigger thickness. In addition, thanks to their perfectly- 
deposited gold layer over the chromium one no problem was presented to observe the exposed polysilicon device layer after the removal of the photoresist. A polysilicon dry etching for 25 seconds using the gold as a mask material defined the chips (Figure 4h). Chips were released by a sacrificial etching of the silicon oxide sacrificial layer in vapors of $49 \% \mathrm{HF}$ for 40 minutes, suspended in $96 \%$ ethanol, centrifuged at $14000 \mathrm{~g}$ for 5 minutes (MiniSpin Plus ${ }^{\circledR}$ ) (Figure 4i). Lastly, chips were collected and stored in eppendorfs for their posterior studies. A study focused on looking at the chip loss during the fabrication process was done counting the chips using a Neubauer Chamber to improve the collection yield. Although 276 million of chips can be simultaneously fabricated in a single wafer; during the release process from the wafer we have concluded that there is a loss of $55 \%$.

\subsection{Material characterization of the chips}

To characterize the three different devices, (polysilicon-gold chips, chromium chips and polysilicon-chromium-gold chips), a combination of a Scanning Electron Microscopy (SEM) and Energy Dispersive-X-ray spectroscopy (EDX) methods was carried out. Devices were pipetted in a $2 \mu \mathrm{L}$-drop and deposited on a clean silicon substrate for inspection. After solvent evaporation the substrates were observed and characterize on a SEM (Carl Zeiss Auriga - Microscope GmbH, Germany). EDX analysis was also performed on pellets sections for a material identification. Images were captured by an Oxford Instruments IncaX-act SEM and the EDX spectrums were obtained using a PentaFET-Precision attached to the SEM equipment. 


\subsection{Orthogonal functionalization of Polysilicon-Chromium-Gold wafers}

Commercial compounds: Absolute ethanol, sulphuric acid (96\%) and acetic acid used were analytical grade. Water used in the experiments was HPLC grade produced by a MilliQ plus system from Millipore (MQ water). 11-(Triethoxysilyl)undecanal (TESUD) and aminoundecyltriethoxysilane (AUTES) was purchased from ABCR, and Mercaptoundecanoate succinimide (NHS) was purchased from Prochimia. Phosphate buffer (PBS), hydrogen peroxide, sodium cyanoborohydride and cysteamine were purchased from Sigma-Aldrich. Texas-Red conjugated WGA (TR-WGA), Fluorescein conjugated Concanavalin (F-ConA), Oregon Green ${ }^{\circledR} 488$ Carboxylic Acid Succinimidyl Ester 6-isomer and BODIPY ${ }^{\circledR}$ 581/591 NHS Ester were purchased from Invitrogen.

In order to evaluate the two detection abilities of the selected proteins (Texas Red-WGA and Fluorescein-ConA) individually and in combination and discard any fluorescence interference between them, a mono-functionalization and a simultaneous bifunctionalization process on polysilicon and gold layers in a single polysiliconchromium-gold wafer was carried out. In this case, the polysilicon main layer was not etched to ensure both functionalization processes in the same surface (Figure 5 - Surface I).

The double functionalization proceeded following several steps: 1) First, polysilicon surface was oxidized through piranha solution $\mathrm{H}_{2} \mathrm{SO}_{4}: \mathrm{H}_{2} \mathrm{O}_{2}(7: 3),(3 \mathrm{~mL})$ for 1 hour, and then, it was rinsed with water. 2) Mercaptoundecanoate-NHS SAM formation on gold: 1 $\mathrm{cm}^{2}$ surface was incubated with an ethanol solution of mercaptoundecanoate-NHS ( $3 \mathrm{~mL}$, $8 \mathrm{mM}$ ) for 3 hours and consequently washed with ethanol. 3) TR-WGA immobilization: The modified surface was immersed in a PBS solution of TR-WGA $(3 \mathrm{~mL}, 35 \mu \mathrm{g} / \mathrm{ml})$ and 
was kept overnight at room temperature. After that, surface was rinsed with PBS (Figure 5 - Surface II). 4) TESUD SAM formation on polysilicon: an ethanol solution of TESUD $(1 \mathrm{~mL}, 135 \mathrm{mM})$ with an acetic buffer $(\mathrm{pH}=5.2)$ was used to immerse another piece of wafer of the already functionalized surface (Surface II) for the double functionalization, and it was incubated for 3 hours at room temperature. Then, it was washed with ethanol and finally dried with air. 5) F-ConA immobilization: The surface was finally immersed in a PBS solution of F-ConA ( $3 \mathrm{~mL}, 35 \mu \mathrm{g} / \mathrm{mL})$ incorporating sodium cyanoborohydride $(500 \mu \mathrm{L}, 5 \mathrm{mM})$ and was kept overnight at $4^{\circ} \mathrm{C}$. Finally, the bi-functionalized surface was washed with PBS and was kept in fresh water (Figure 5 - Surface III). Surface II and Surface III were kept for an individual and in combination protein fluorescence activity validation. Finally, the two last steps were 6) TESUD SAM formation on polysilicon: an ethanol solution of TESUD $(1 \mathrm{~mL}, 135 \mathrm{mM})$ with an acetic buffer $(\mathrm{pH}=5.2)$ was used to immerse a non-functionalized surface (Surface I) for the silicon functionalization, and it was incubated for 3 hours at room temperature. Then, it was washed with ethanol and finally dried with air. 7) F-ConA immobilization: The surface was finally immersed in a PBS solution of F-ConA ( $3 \mathrm{~mL}, 35 \mu \mathrm{g} / \mathrm{mL}$ ) incorporating sodium cyanoborohydride (500 $\mu \mathrm{L}, 5 \mathrm{mM}$ ) and was kept overnight at $4^{\circ} \mathrm{C}$ (Figure 5 - Surface IV). In this case, the hexadecanethiol was used instead of the mercaptoundecanoate-NHS in order to coat the gold, besides the fact that TR-WGA was not added.

2.6. Orthogonal functionalization methods of suspended Polysilicon-Chromium-Gold chips 
After observing that the detection abilities of the selected proteins during simultaneous functionalization processes were not any how affected, the Texas Red-WGA and Fluorescein-ConA double functionalization protocol was adjusted for the functionalization of the Polysilicon-Chromium-Gold released chips. First, polysilicon was oxidized through piranha solution $\mathrm{H}_{2} \mathrm{SO}_{4}: \mathrm{H}_{2} \mathrm{O}_{2}(7: 3)$, (1 mL) for 1 hour. Next, chips were centrifuged (13000 rpm, $5 \mathrm{~min})$ and they were washed adding MQ water ( $1 \mathrm{~mL} \mathrm{x}$ 3), replacing the supernatant in every centrifugation with clean water (Figure 6a and b). Chips were incubated with an ethanol solution of mercaptoundecanoate-NHS ( $1 \mathrm{~mL}, 8$ $\mathrm{mM})$ for 3 hours and consequently washed using centrifugation with ethanol ( $1 \mathrm{~mL} \times 3)$ (Figure 6c). A PBS solution of TR-WGA $(1 \mathrm{~mL}, 35 \mu \mathrm{g} / \mathrm{ml})$ was added to the chips suspension and was kept overnight at room temperature. After that, chips were washed using centrifugation with PBS (1 mL x 3) (Figure 6d). An ethanol solution of TESUD (1 $\mathrm{mL}, 135 \mathrm{mM})$ with an acetic buffer $(\mathrm{pH}=5.2)$ was added to the chips suspension, and they were incubated for 3 hours at room temperature. Then, they were washed with ethanol using centrifugation $(1 \mathrm{~mL} \times 3)$ and finally dried with air (Figure 6e). A PBS solution of F-ConA ( $1 \mathrm{~mL}, 35 \mu \mathrm{g} / \mathrm{mL})$ incorporating sodium cyanoborohydride $(100 \mu \mathrm{L}$, $5 \mathrm{mM}$ ) was added to the chips suspension and they were kept overnight at $4^{\circ} \mathrm{C}$ (Figure 6f). Finally, the biofunctionalized chips were washed using centrifugation with PBS (1 $\mathrm{mL}$ x 3) and they were kept in fresh water. Fluorescence images were obtained with a Leica DMIRB inverted fluorescence microscope.

For cell internalization studies chips were functionalized using the same procedure described above but with two different dyes instead of the proteins. Oregon green was immobilized on the gold using cysteamine and Bodipy 581/591 was immobilized on polysilicon by the aminoundecyltriethoxysilane. 
2.7. Cell internalization and cytotoxicity assays of non-functionalized Chromium and Polysilicon-Chromium-Gold chips

In order to study the impact of the presence of chromium or polysilicon-chromium-gold chips in cell toxicity, a first cell internalization study of non-functionalized chips in human macrophages was carried out.

To study chips internalization, THP-1 cells (ATCC) were seeded in 24-well dishes at a density of $10^{5}$ cells/well and differentiated into macrophages using $0.16 \mu \mathrm{M}$ phorbol 12 myristate 13-acetate (Sigma-Aldrich) in RPMI 1640 (Gibco) supplemented with $20 \%$ Fetal Bovine Serum (PAA) for 72h. After cell differentiation, medium was replaced by fresh culture medium containing chips at a 2:1 chips/cell ratio. At days 1, 3 and 7, viability was assessed by a differential staining for live and dead cells using the LIVE/DEAD ${ }^{\circledR}$ Viability/Cytotoxicity Kit for mammalian cells (L3224; Life Technologies). Briefly, the kit consists of calcein acetomethoxy, which can diffuse through the cell membrane and is converted to highly green fluorescent calcein by intracellular esterases of living cells, and ethidium homodimer-1, which can only penetrate cells with a damaged plasma membrane reaching the nucleus and labeling the DNA of dead cells with red fluorescence. Labeled cells were analyzed under an inverted fluorescence microscope (Olympus IX71, Germany). For each treatment, three independent experiments were performed, counting 250 cells for each one. In addition and in parallel, at days 1, 3 and 7, after the addition of the chips, cells were fixed in $2.5 \%$ glutaraldehyde in cacodylate buffer, dehydrated in an ethanol series, dried by a 15 min treatment with hexamethyldisilazane and observed under a scanning electron microscope (Carl Zeiss Merlin - Microscope GmbH, Germany).

To determine the localization of the chip (i.e., inside the cells or attached to their plasma membrane), cells were seeded at a density of $10^{5}$ cells/dish on $35 \mathrm{~mm}$-diameter glass- 
bottom coverslip dishes (MatTek Corp, Ashland, MA) and induced to differentiate to macrophages as explained before. After cell differentiation, medium was replaced by fresh culture medium containing chips at a 2:1 chips/cell ratio. After $24 \mathrm{~h}$, cells were stained with TR-WGA (10 $\mu \mathrm{g} / \mathrm{mL}$; Life Technologies) and counterstained with Hoescht $33258(1 \mu \mathrm{g} / \mathrm{mL}$; Sigma-Aldrich), to visualize the plasma membrane and the nucleus, respectively. Samples were then examined under a Confocal Laser Scanning Microscope (CLSM: Fluoview ${ }^{\circledR}$ FV1000; Olympus Corp, Tokyo, Japan) using a $63 \times$ oil immersion objective, where $x-y-z$ sequential acquisition was achieved and orthogonal projections of the stacks were evaluated to determine the location of the chip within the cell. For the image analyses, the FV10-ASW Application Software (Ver. 01.07c; Olympus) was used. Statistical analyses were done using SPSS Statistics Ver. 19.0 software (IBM Oregon, USA). Normality and homoscedasticity was tested using the Kolmogorov-Smirnov and Levene tests. A t-test for repeated measures was run using the presence/absence of microparticles as inter-subject factor and the incubation time as intra-subject factor. The level of significance was set at $\mathrm{P}<0.05$.

\subsection{Cell internalization of bi-functionalized Polysilicon-Chromium-Gold chips}

To study the internalization of bi-functional multi-material platforms, cells were seeded at a density of 105 cells/dish on $35 \mathrm{~mm}$-diameter glass-bottom coverslip dishes (MatTek Corp, Ashland, MA) and induced to differentiate to macrophages as explained before. After $24 \mathrm{~h}$ of being in contact with bi-functional chips, cells were stained with CellMask $^{\mathrm{TM}}$ Deep Red plasma membrane stain $(10 \mu \mathrm{g} / \mathrm{mL}$; Life Tech $\neg$ nologies $)$ to visualize the plasma membrane. Samples were then examined under a Confocal Laser Scanning Microscope (CLSM: Fluoview® FV1000; Olympus Corp, Tokyo, Japan) using a $63 \times$ oil immersion objective, where $x-y-z$ sequential acquisition was achieved and 
orthogonal projections of the stacks were evaluated to determine the location of the chip inside the cell.

\section{Results and discussions}

\subsection{Polysilicon-Gold chips fabrication results}

As it can be observed using scanning electron microscopy, the polysilicon etching was not properly performed at the wafer level (Figure 7a). This could be related to the fact that a non-defined gold layer was deposited because of the well-known bad and weak adherence between gold and silicon [35-37], as gold has acted as a mask during the polysilicon dry etching and homogenous edges were necessary to clearly define the devices. After the wafer release the bad adherence of gold over the silicon layer was highly demonstrated, this fact can be seen on figure $7 \mathrm{~b}$ and $\mathrm{c}$, where the polysilicon and gold layers were completely separated. All this means that non-robust devices have been obtained. In order to circumvent the difficulty of attaching gold layers to the polysilicon platforms, an intermediated adherent-layer should be deposited in between to improve the adhesion. For instance, titanium and chromium are common materials used as efficient adherents in microelectronics and microsystems technologies. However, in this case, titanium was not suitable due to its non-resistant capability to be etched by concentrated hydrofluoric acid, a key step in the release process of our devices. Therefore, chromium was selected as an adherent material because of its HF-resistance. As a result, metallic chromium cell viability should be evaluated first, and accordingly, chromium microchips were fabricated and their bio-compatibility was analyzed.

\subsection{Chromium chips fabrication results}


Figure 8a shows a SEM image of a $100 \mathrm{~mm} \varnothing$ p-type silicon wafer with $100 \mathrm{~nm}$-thick chromium chips on its surface, demonstrating that thin mono-material chips can be successfully fabricated at the wafer level. Also, Figure $8 \mathrm{~b}$ shows several released chromium chips after the sacrificial etching; these devices showed high robustness, homogeneous surface and a perfectly defined shape, in despite of their small thickness. Energy-dispersive X-ray (EDX) analysis was performed for material characterization (Figure $8 \mathrm{~b}$ and $\mathrm{c}$ ). In the graph included in Figure 8c, the characteristic peak of chromium signal at $0.573 \mathrm{KeV}$ can be observed.

\subsection{Cell viability and internalization of the Chromium chips}

Observation under optical light microscopy and CLSM showed that chromium chips were efficiently internalized by cells. Several macrophages with chromium chips can be observed in figure 9a, all of them, carrying or not a chip, showed green fluorescence (live cells) under the fluorescent microscope (Figure 9b). SEM studies confirmed the normal shape and membrane integrity of macrophages carrying a chromium chip (Figure 9c). Some cells were analyzed by CLSM, where the orthogonal projections of the z-stack reconstruction of consecutive focal planes $(0.5 \mu \mathrm{m}$ each $)$ confirmed the localization of the chips inside the cells (Figure 9d and e, respectively). Finally, no statistically significant differences were found between macrophage cultures incubated with chromium chips during 1, 3 and 7 days and control cultures (Figure 9f), demonstrating that the exposure to chromium chips did not interfere in cell viability. Thus, taken together these results we can conclude that our chips do not affect cell viability. This fact has been well documented by researchers worldwide, as health hazards associated with exposure to chromium are dependent on its oxidation state. For instance, the toxicity and carcinogenic properties of chromium (VI) are known for a long time [39]. However, water insoluble chromium metal (our case of study) is not considered as a toxic material [40]. Accordingly 
to these results, the use of chromium as adherent layer between polysilicon and gold was probed.

\subsection{Polysilicon-Chromium-Gold chips fabrication results}

Finally, polysilicon-chromium-gold devices with a high versatility and robustness were manufactured. Polysilicon-chromium-gold chips with lateral dimensions of $3 \mu \mathrm{m}$ and with a total thickness of $530 \mathrm{~nm}$ (Polysilicon: $400 \mathrm{~nm}$ - Chromium: $30 \mathrm{~nm}$ - Gold: 100 $\mathrm{nm}$, respectively) were successfully fabricated. A SEM image of these chips on the wafer (Figure 10a and inset is a zoom image) shows that, in this case, the polysilicon dry etching was perfectly performed, as the gold layer showed a homogenous defined shape. Here, the gold layer was deposited over the chromium layer achieving a good adhesion and ensuring a very robust device. In figure $10 \mathrm{~b}$ it can be seen the chips after their wafer release, showing their well-defined shape and homogenous surface. EDX analysis was also performed for thorough material identification. The black and red encircled areas in Figure 10c show the point where the EDX analyses were carried out. The characteristic silicon and gold X-Ray signals were showed at $1.739 \mathrm{KeV}$ and $2.120 \mathrm{KeV}$, respectively (Figure 10d). However, if the X-Ray beam fell on the polysilicon layer or on the gold layer, the chromium signal was not easily revealed, as their small thickness (only $30 \mathrm{~nm}$ ) was probably masked by the silicon and gold higher signals. Nevertheless, if the EDX spot fell in the middle of the multi-material layer (Figure 10e) when the chip is tilted, we can easily recognize the chromium signal at $0.573 \mathrm{KeV}$, along with the silicon and gold signals at $1.739 \mathrm{KeV}$ and $2.120 \mathrm{KeV}$, respectively (Figure 10f). Accordingly to these results highly defined polysilicon- gold chips were manufactured using chromium as adherent layer. 


\subsection{Polysilicon-Chromium-Gold wafers and suspended chips functionalization and}

\section{fluorescence characterization}

As probe of concept, multi-material polysilicon-chromium-gold wafers and chips were functionalized using orthogonal chemistry. The different chemical reactivity of the gold and the polysilicon substrates prompted us to combine the chemistry of thiols to coat the gold surface [41], and alcoxysilane derivatives [42] to functionalize the polysilicon side. Both the selected thiol and silanes self-assemble efficiently on surfaces, and incorporate functional groups capable of establishing covalent bonds with biomolecules, to furnish the microchips with robust bi-functionality. Furthermore, in order to prove the immobilization of different proteins and discard protein interaction defects as aggregation, two different fluorescently labelled lectins were immobilized [43] on the gold as well as on the polysilicon surfaces. Lectins are proteins that are characteristic for their capacity to interact with different carbohydrates, playing an important role in cellular recognition [44]. In fact, our group has shown previously their use for the functionalization of monofunctional polysilicon barcodes for cell tagging. [14-17] It is worth mentioning that the order of the biofuctionalization steps is highly relevant, as a surface activation of the polysilicon layer is needed to be done first, because otherwise any organic or biological element will be removed from the layer. After that, the gold layer needs to be covered before the polysilicon one to avoid unspecific chemisorptions.

Thus, double biofunctionalization was achieved in four steps. First, TR-WGA was immobilized on the gold surface, after the formation of a self-assembled monolayer using mercaptoundecanoate-NHS. Next, the silane TESUD was self-assembled on the polysilicon side, and subsequently Fluorescein green Concanavalin (F-ConA) was covalently immobilized, under reductive amination conditions. Bi-functionalization was attempted in polysilicon-chromium-gold wafers before to use the chips, in order to 
stablish the optimal procedure for the immobilization of both labelled proteins. Figure 11 shows a bi-functional wafer in which the gold film showed red fluorescence while the polysilicon layer exhibits green fluorescence.

After the surface functionalization processes, fluorescence microscopy was used to characterize the released double biofunctionalized chips. Figure 12 shows several biofunctionalized chips in which their fluorescence emission is dependent on the fluorophores tagging the immobilized proteins (Texas-Red for WGA red or Fluorescein for ConA green). In addition, whether the chips show fluorescence or not depends on the functionalized substrate that is located in the upper part of the standing microchip. It is clearly seen that if the gold substrate is at the top, red fluorescence is detected, whereas if the polysilicon side is at the top, the green fluorescence is observed (Figure 12a-c). Moreover, figures $12 \mathrm{~d}-\mathrm{f}$ show that the fluorescence emission can be also detected even when chips were partially overlap. These images evidence the homogenous immobilization of both lectins onto the polysilicon-chromium-gold chips, and prove the possibility of preparing bi-functional microchips. Furthermore, characterization by fluorescence microscopy, using the wafers as well as the chips, has not shown any interference in the fluorescence emission of the fluorescently labelled proteins, which seems to indicate that no interaction between functional layers is occurring. In addition, the orthogonal chemical functionalization and the presence of eleven carbon atoms in the SAM, would avoid the interaction between the two immobilized biomolecules.

In addition, a study focused on looking at the chip loss after the several steps of the functionalization process using a Neubauer Chamber was also done. As we explained before, during the chip release process we concluded that there was a chip loss of 55\%. Now, after the functionalization processes we should add a loss of $24 \%$. Although there was an increasing loss after the performance of the different processes, the number of 
fabricated chips was high enough to accomplish their purpose as multi-material platforms for intracellular biosensing.

Once the double functionalization of the bi-functional chips was achieved, the internalization of the particles was aimed. In this case, two different dyes commonly used in cell biology were immobilized instead of the two lectins, because lectins could impede the chips internalization due to their high affinity for the extracellular cell membranes. Thus, Oregon green (green emission) and Bodipy (red emission) were used to afford the visualization of their corresponding fluorescence onto the chips inside the cells.

\subsection{Cell viability and internalization of non-functionalized Polysilicon-Chromium-Gold} chips

In figure $13 \mathrm{a}$ and $\mathrm{b}$ it can be seen that most of the cells were alive (green fluorescence) when were observed under the fluorescent microscope and that scarce death cells (red cells) can be observed. In order to determine the location of these chips (i.e. if they are attached to the cell membrane or inside the cell), scanning electron microscopy analysis was carried out. SEM images showed that the multi-material chips were successfully phagocyted by macrophages. In figure $13 \mathrm{c}$ a macrophage in the process of engulfing a chip, by membrane evaginations, can be observed and some phagocyted chips can also be seen. In addition, cells exposed to chips conserved their normal morphology and membrane integrity. Finally, some cells were analyzed by CLSM to confirm cell internalization (Figure 13d and e). Approximately, $45 \%$ of the macrophages had internalized at least one chip. Even the phagocytosis of several chips (in figure $11 \mathrm{~d}$ and e several macrophages with 3 or more phagocyted chips can be observed) did not interfere on cell viability. No significant differences were found between macrophage cultures incubated with polysilicon-chromium-gold chips during 1, 3 and 7 days and control 
cultures (Figure 13f), indicating that the incubation of macrophages in presence of polysilicon-chromium-gold chips did not affect significantly cell viability. The reduction in macrophage viability can be attributed to the normal behavior of these cells after differentiation, which attach to the substrate, stop proliferating, and finally die and not to the presence of the chips [45].

3.7. Cell viability and internalization of bi-functionalized Polysilicon-Chromium-Gold chips

After probing that non-functionalized multi-material chips are internalized by macrophages and are not cytotoxic, a cell internalization study of bi-functional polysilicon-chromium-gold chips as intracellular platforms was performed. In figure 14a a conceptual representation of several functional polysilicon-chromium-gold chips inside a living macrophage is shown. Red or green fluorescence can be observed depending on whether the chips are facing downwards or upwards. In this case, red fluorescence signal belongs to the functionalized gold layer (Figure 14b), while the green fluorescence signal belongs to the functionalized polysilicon layer (Figure 14c).

\section{Conclusions}

In this paper the fabrication of bi-functional polysilicon-chromium-gold chips for intracellular applications are presented. These devices can be successfully fabricated using semiconductor technologies based on photolithographic techniques. Polysilicon and gold as device materials were selected because of their high versatility and wellknown orthogonal chemistry. However, the well-known weak adhesion of metallic gold 
to silicon substrates [35-37] limits its use in this type of devices. To circumvent this fabrication issue, a chromium interlayer was selected to be deposited in between, due to its well-known adherent properties and its high resistance to HF-wet etchings. As a previous step and to confirm the possibility of using metallic chromium to manufacture cell-internalizable devices, chips entirely made of this material were manufactured and their cell-viability was evaluated and confirmed. In the light of these results, an ultra-thin (30 nm) chromium interlayer was deposited in between polysilicon and gold device layers causing a notably increased in the adherence. Finally, robust and perfectly defined polysilicon-chromium-gold chips were fabricated. In order to provide the multi-material devices with bi-functional capabilities, two different lectins were immobilized. This doubled functionalization was successfully performed on both wafers and suspended chips using orthogonal chemistry, in order to discern if the detection abilities of the selected proteins were affected by the simultaneous functionalization processes. Fluoresceinamine labelled Concanavalin and the Texas red labelled Wheat Germ Agglutinin were used to selectively bi-functionalize polysilicon and gold layers through self-assembled monolayers. Fluorescence microscopy was used for unambiguous characterization of the bi-functionalized chips. Internalization and cell-viability tests with non-functionalized chips were also performed to ensure their cell viability for future bioapplications. The obtained results showed high cell viability when they were compared with control cell cultures. Eventually, a cell internalization process with bi-functionalized multi-material chips was successfully performed.

In summary, in this paper we provide a simple but effective method to manufacture polysilicon-chromium-gold devices as bi-functional cell-viable platforms for future biosensors and intracellular drug delivery systems, specifically we are actually involved in different combinations of an apoptosis inductor and a ROS sensor. 


\section{Acknowledgements}

This study was financed by the Spanish government through project MINAHE4 (TEC2011-29140-C03). S. D. thanks the MICINN for her predoctoral grant. And T. P. thanks the Generalitat de Catalunya for her predoctoral grant. The authors also thank the cleanroom staff of IMB-CNM for fabrication of the chips.

\section{Highlights}

We show the efficiency of metal chromium as adherent material.

We ensure cell-internalization and viability of chromium devices.

We fabricate highly versatile and robust PolySi-Cr-Au chips.

We doubly-functionalize PolySi-Cr-Au chips using two different fluorescent proteins.

We show the cellular uptake of the polysilicon-chromium-gold chips without affecting the cell viability.

Corresponding author at: Instituto de Microelectrónica de Barcelona, IMB-CNM (CSIC), Campus UAB, 08193, Cerdanyola, Barcelona, Spain. Tel.: +34 9359477 00; fax: (+34) 9358002 67.E-mail address: joseantonio.plaza@imb-cnm.csic.es 


\section{References}

[1] J. El-Ali, P. K. Sorger, K. F. Jensen, Cell on a Chip, Nature 442 (2006) 403-411.

[2] D. B. Weibel, W. R. DiLuzio, G. M. Whitesides, Microfabrication meets microbiology, Nature 5 (2007) 209-218.

[3] A. K. Shalek, J. T. Robinson, E. S. Karp, J. S. Lee, D-R. Ahn, M. H. Yoon, A. Sutton, M. Jorgolli, R. S. Gertner, T. S. Gujral, G. MacBeath, E. G. Yang, H. Park, Vertical silicon nanowires as a universal platform for delivering biomolecules into living cells, PNAS, 107 (2010) 1870-1875.

[4] Y. Hu, D. H. Fine, E. Tasciotti, A. Bouamrani, M. Ferrari, Nanodevices in diagnostics, WIREs Nanomedicine and Nanobiotechnology, 3 (2011) 11-32.

[5] K. R. Kam, T. A. Desai, Nano- and microfabrication for overcoming drug delivery challenges, J. Mater. Chem. B, 1 (2013) 1878-1884.

[6] S. E. Cross, Y-S. Jin, J. Rao, J. K. Gimzewski, Nanomechanical analysis of cells from cancer patients, Nature Nanotech. 2 (2007) 780-783.

[7] S. Ferrati, A. Mack, C. Chiappini, X. Liu, A. J. Bean, M. Ferrari, R. E. Serda, Intracellular trafficking of silicon particles and logic-embedded vectors, Nanoscale, 2 (2010) 1512-1520.

[8] R. E. Serda, J. Gu, R. C. Bhavane, X. W Liu, C. Chiappini, P. Decuzzi, M. Ferrari, The association of silicon microparticles with endothelial cells in drug delivery to the vasculature, Biomaterials 30 (2009) 2440-2448.

[9] A. Fulati, S. M. U. Ali, M. H. Asif, N. H. Alvia, M. Willandera, C. Brännmarkb, P. Strålfors, S. I. Börjessonb, F. Elinderb, B. Danielssond, An intracellular glucose biosensor based on nanoflake ZnO, Sensors and Actuators B, 150 (2010) 673-680.

[10] T. F. Otero, J. G. Martinez, Biomimetic intracellular matrix (ICM) materials, properties and functions. Full integration of actuators and sensors, J. Mater. Chem. B, 1 (2013) 26-38.

[11] E. Fernández-Rosas, R. Gómez-Martínez, E. Ibáñez, L. Barrios, M. Duch, J. Esteve, C. Nogués, J. A. Plaza, Intracellular polysilicon barcodes for cell tracking, Small 5 (2009) 2433-2439.

[12] R. Gómez-Martínez, A. M. Hernández-Pinto, M. Duch, P. Vázquez, K. Zinoviev, E. J. de la Rosa, J. Esteve, T. Suárez, J. A. Plaza, Silicon chips detect intracellular pressure changes in living cells, Nature Nanotech. 8 (2013) 517-521.

[13] R. Gómez-Martínez, P. Vázquez, M. Duch, A. Muriano, D. Pinacho, N. Sanvicens, F. Sánchez-Baeza, P. Boya, E. J. de la Rosa, J. Esteve, T. Suárez, J. A. Plaza, Intracellular silicon chips in living cells, Small 4 (2010) 499-502. 
[14] O. Penon, S. Novo, S. Durán, E. Ibañez, C. Nogués, J. Samitier, M. Duch, J. A. Plaza, L. Pérez-García, Efficient biofunctionalization of polysilicon barcodes for adhesion to the zona pellucida of mouse embryos, Bioconjugate Chem. 23 (2012) 137-141.

[15] S. Novo, R. Morató, O. Penon, S. Durán, L. Barrios, C. Nogués, R. Gómez-Martínez, L. Pérez-García, J.A. Plaza, E. Ibáñez, T. Mogas, Identification of bovine embryos cultured in groups by attachment of barcodes to the zona pellucida, Reprod. Fertil. Dev. 25 (2013) 218-219.

[16] S. Novo, O. Penon, L. Barrios, C. Nogués, J. Santaló, S. Durán, R. Gómez-Martínez, J. Samitier, J. A. Plaza, L. Pérez-García, E. Ibáñez, Direct embryo tagging and identification system by attachment of biofunctionalized polysilicon barcodes to the zona pellucida of mouse embryos, Hum. Reprod. 286 (2013) 1519-1527.

[17] S. Novo, C. Nogués, O. Penon, L. Barrios, J. Santaló, Rodrigo Gómez-Martínez, J. Esteve, A. Errachid, J. A. Plaza, L. Pérez-García, E. Ibáñez, Barcode tagging of human oocytes and embryos to prevent mix-ups in assisted reproduction technologies, Hum. Reprod. 291 (2014) 18-28.

[18] S. Kelkar, T. M. Reineke, Bioconjugate Chemistry 22 (2011) 1879-1903.

[19] O. Crespo-Biel, B. J. Ravoo, D. N. Reinhoudt, J. Huskens, J. Mater. Chem. 16 (2006) 3997-4021.

[20] N. Patel, M. C. Davies, M. Hartshorne, R. J. Heaton, C. J. Roberts, S. J. B. Tendler, P. M. Williams, Langmuir 13 (1997) 6485-6490

[21] P. S. Cremer, JACS Select 11: Surface Patterning, More than just Scratching the Surface, J. Am. Chem. Soc. 133 (2011) 167-169.

[22] L. Nebhani, C. Barner-Kowollik, Orthogonal transformations on solid substrates: efficient avenues to surface modification, Adv. Mater. 21 (2009) 3442-3468.

[23] H.Y. Xie, C. Zuo, Y. Liu, Z. L. Zhang, D. W. Pang, X. L. Li, J. P. Gong, C. Dickinson, W. Zhou, Cell-Targeting Multifunctional Nanospheres with both Fluorescence and Magnetism, Small 5 (2005) 506-509.

[24] C. Kaewsaneha, P. Tangboriboonrat, D. Polpanich, M. Eissa, A. Elaissari, Janus Colloidal Particles: Preparation, Properties, and Biomedical Applications, ACS Appl. Mater. Interfaces 5 (2013) 1857-1869.

[25] M. Veiseh, M. H. Zareie, M. Zhang, Highly Selective Protein Patterning on GoldSilicon substrates for biosensor applications, Langmuir 18 (2002) 6671-6678.

[26] E. Briand, V. Humblot, J. Landoulsi, S. Petronis, C. M. Pradier, B. Kasemo, S. Svedhem, Chemical Modifications of $\mathrm{Au} / \mathrm{SiO} 2$ template substrates for patterned biofunctional surfaces, Langmuir 27 (2011) 678-685. 
[27] J. L. Tang, K. Schoenwald, D. Potter, D. White, T. Sulchek, Langmuir 28 (2012) 10033-10039.

[28] M. Nordström, A. Johansson, E. Sánchez Nogueron, M. Calleja, A. Boisen, Investigation of the bond strength between the photo-sensitive polymer SU-8 and gold. Microelectron. Eng. 78 (2005) 152-157.

[29] O. Penon, D. Siapkas, S. Novo, S. Durán, G. Oncins, A. Errachid, L. Barrios, C. Nogués, M. Duch, J. A. Plaza, L. Pérez-García, Optimized immobilization of lectins using self-assembled monolayers on polysilicon encoded materials for cell tagging, Colloids Surf. B. 116 (2014) 104-113.

[30] J. Fan, P. K. Chu, Group IV Nanoparticles: Synthesis, properties, and biological applications, Small 6 (2010) 2080-2098.

[31] T. Shirman, R. Kaminker, D. Freeman, M. E. van der Boom, Halogen-bonding mediated Stepwise assembly of gold nanoparticles onto planar surfaces, ACS Nano, 5 (2011) 6553-6563.

[32] P. Pyykkö, Theoretical Chemistry of Gold, Angew. Chem. 43 (2004) 4412-4456.

[33] E. Fernández-Rosas, R. Gómez-Martínez, E. Ibañez, L. Barrios, M. Duch, J. Esteve, J. A. Plaza, C. Nogués. Biomed. Microdev. 12 (2010) 371-379.

[34] D. M.Wilson, S. Hoyt, J. Janata, K. Booksh, L. Obando, IEEE Sens. J. 1 (2001) 256274.

[35] M. A. George, W.S. Glaunsinger, T. Thundat, S. M. Lindsay, Thin Solid Films 189 (1990) 59-72.

[36] R. Audino, G. Destefanis, F. Gorgellino, E. Pollino, S. Tamagno, Thin Solid Films 36 (1976) 343-347.

[37] K. D. Kang, R. R. Burgess, M. G. Coleman, J. G. Keil, IEEE Trans. Electron. Devices 16 (1969) 356-360.

[38] N. R. Moody, D. P. Adams, D. Medlin; T. Headley; N. Yang; A. Volinsky. Int. J. Fract. 119 (2003) 407-419.

[39] D. G. Barceloux, J. Toxicol. Clin. Toxicol. 372 (1999) 173-194.

[40] L. Kanerva, A. Aitio, European Journal of Dermatology. 72 (1997) 79-84.

[41] J. C. Love, L. A. Estroff, J. K. Kriebel, R. Nuzz, G. M. Whitesides, Self-assembled monolayers of thiolates on metals as a form of nanotechnology, Chem. Rev. 105 (2005) 1103-1170. 
[42] C. Haensch, S. Hoeppener, U. Schubert, Chemical modification of self-assembled silane based monolayers by surfaces reactions, Chem. Soc. Rev. 39 (2010) 23232334.

[43] L. S. Wong, F. Khan, J. Micklefield, Selective covalent protein immobilization: strategies and applications, Chemical Reviews, 109 (2009) 4025-4053.

[44] H. Lis, N. Sharon, Lectins: Carbohydrate-Specific proteins that mediate cellular recognition, Chemical Reviews 982 (1998) 637-674.

[45] S. Tsuchiya, Y. Kobayashi, Y. Goto, H. Okumura, S. Nakae,T. Konno, K. Tada, Cancer Res. Induction of maturation in cultured human monocytic leukemia cells by a phorbol diester. 42 (1982) 1530-1536. 
Technological development of intracellular polysilicon-chromium-gold chips for orthogonal chemical functionalization

Figures and captions 


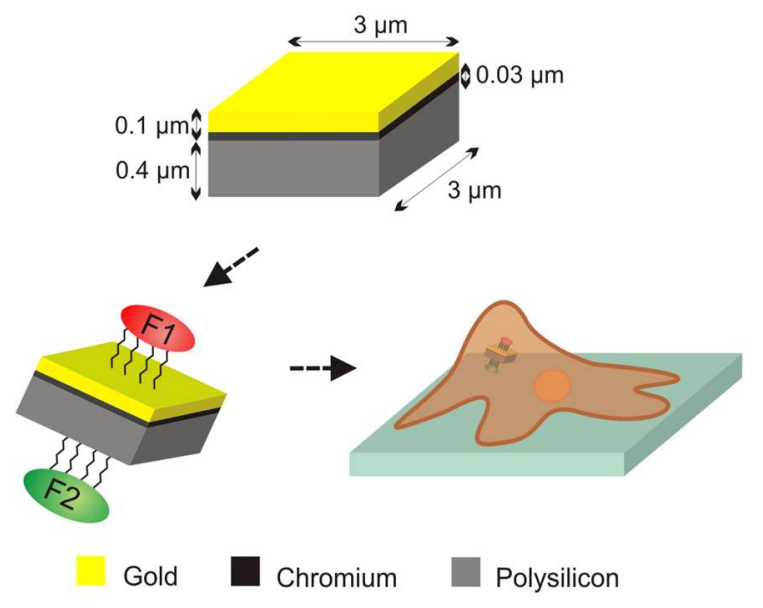

Figure1. Conceptual representation of bi-functional polysilicon-chromium-gold chips for intracellular applications. Polysilicon and gold layers can be selectively bi-functionalized with two different fluorescently labelled proteins, F1 and F2, giving them a highly specific double functionality. A chromium inter-layer is deposited in between as an efficient adherent layer. In addition, these chips can be internalized inside living cells. 
a

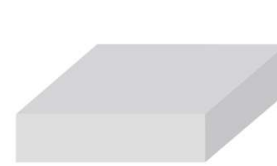

e

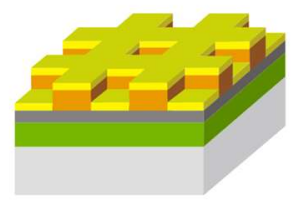

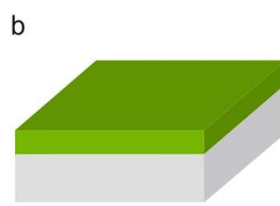

f

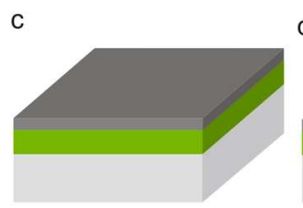

g h

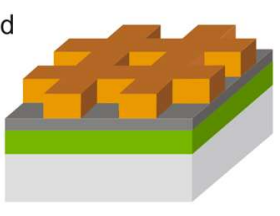

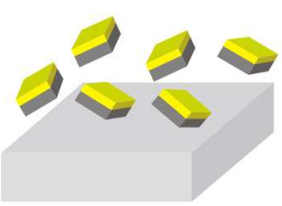

Silicon

Silicon oxide

Polysilicon

Photoresist

Gold

Figure 2. Fabrication process of the polysilicon-gold chips. a) Devices were fabricated starting from a silicon substrate. b) A $1 \mu \mathrm{m}$ silicon oxide was deposited as a sacrificial layer and c) a 400 $\mathrm{nm}$ polysilicon layer were deposited as a device layer. d) $2 \mu \mathrm{m}$-thick photoresist layer was spun and exposed to UV light to define the devices. e) A $100 \mathrm{~nm}$-thick gold layer was deposited by sputtering method as a second-device layer. Then, f) a lift-off process was performed. g) The polysilicon layer was pattern using the gold layer as a mask to define the chips. Finally, h) the polysilicon-gold devices were released in HF vapors and suspended in ethanol. 


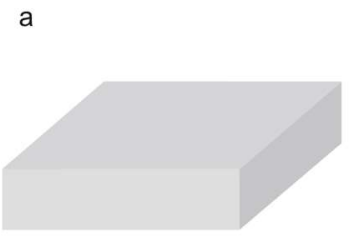

e

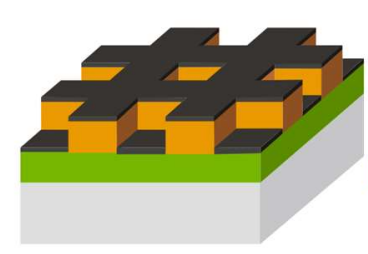

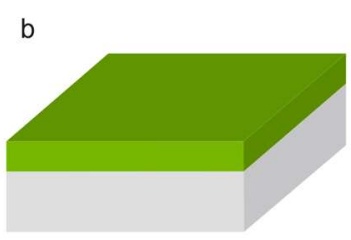

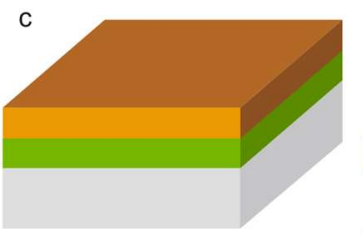

g

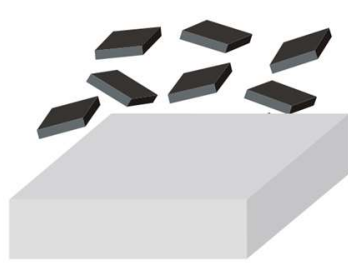

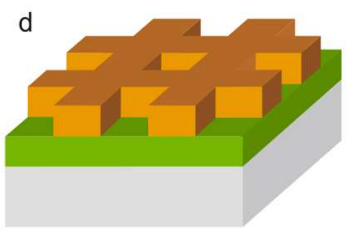

Silicon

Silicon oxide

Photoresist

Chromium

Figure 3. Fabrication process of the chromium chips. a) Starting from a silicon substrate chromium chips were fabricated. b) A $1 \mu \mathrm{m}$ silicon oxide was deposited as a sacrificial layer and c) a $2 \mu \mathrm{m}$ photoresist layer was spun over the surface. d) The photoresist layer was exposed to UV light to define the devices. e) A $100 \mathrm{~nm}$-thick chromium layer was deposited by sputtering process and a f) lift-off process was performed. Finally, g) the chromium devices were released from the wafer in HF vapors and suspended in ethanol. 

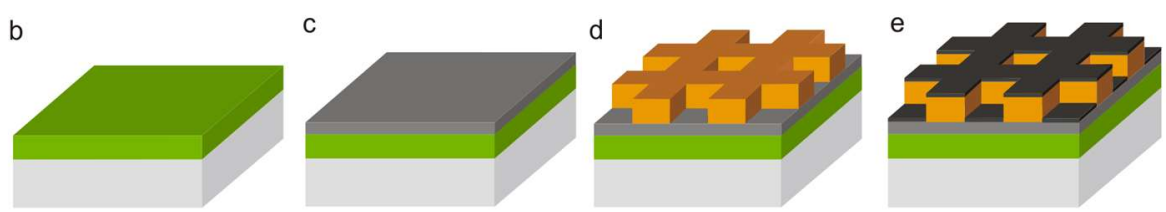

f

g

h

i

Silicon
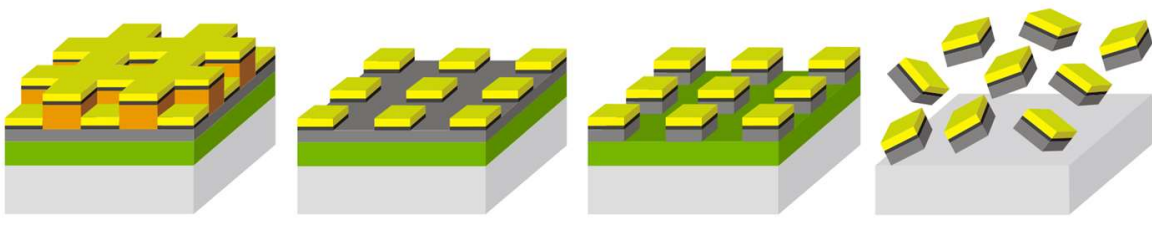

Silicon oxide

Polysilicon

Photoresist

Chromium

Gold

Figure 4. Fabrication process of the polysilicon-chromium-gold chips. a) Chips were fabricated using a silicon wafer as a starting material. b) A $1 \mu \mathrm{m}$ silicon oxide as a sacrificial layer and c) a $400 \mathrm{~nm}$ polysilicon as a first device-layer was deposited. d) A photoresist layer was spun and exposed to UV light to define the dimensions of the devices. e) A $30 \mathrm{~nm}$-thick chromium and f) a $100 \mathrm{~nm}$-thick gold layers were deposited by sputtering process as an adherent and second device-layer, respectively. g) A lift-off process was performed and h) the polysilicon layer was pattern using the chromium and gold layer as a masks. Finally, i) the polysilicon-chromium-gold chips were released in HF vapors and suspended in ethanol. 


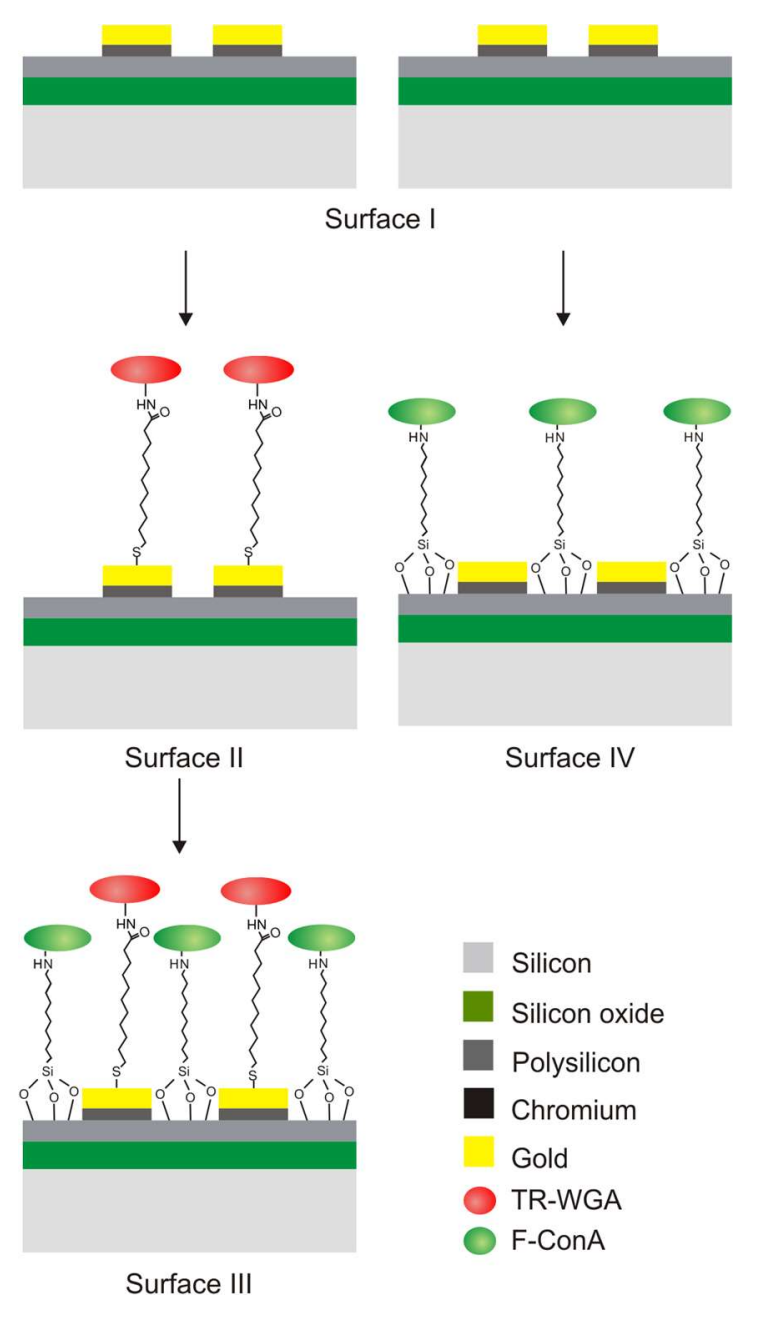

Figure 5. Stepwise formation of mono-functionalization and double biofunctionalization process on polysilicon-chromium-gold wafers. a) Polysilicon-chromium-gold wafer, b) mercaptoundecanoate-NHS SAM formation on gold layer, c) TR-WGA immobilization on the already functionalized wafer, d) TESUD-SAM formation on polysilicon layer. 
f

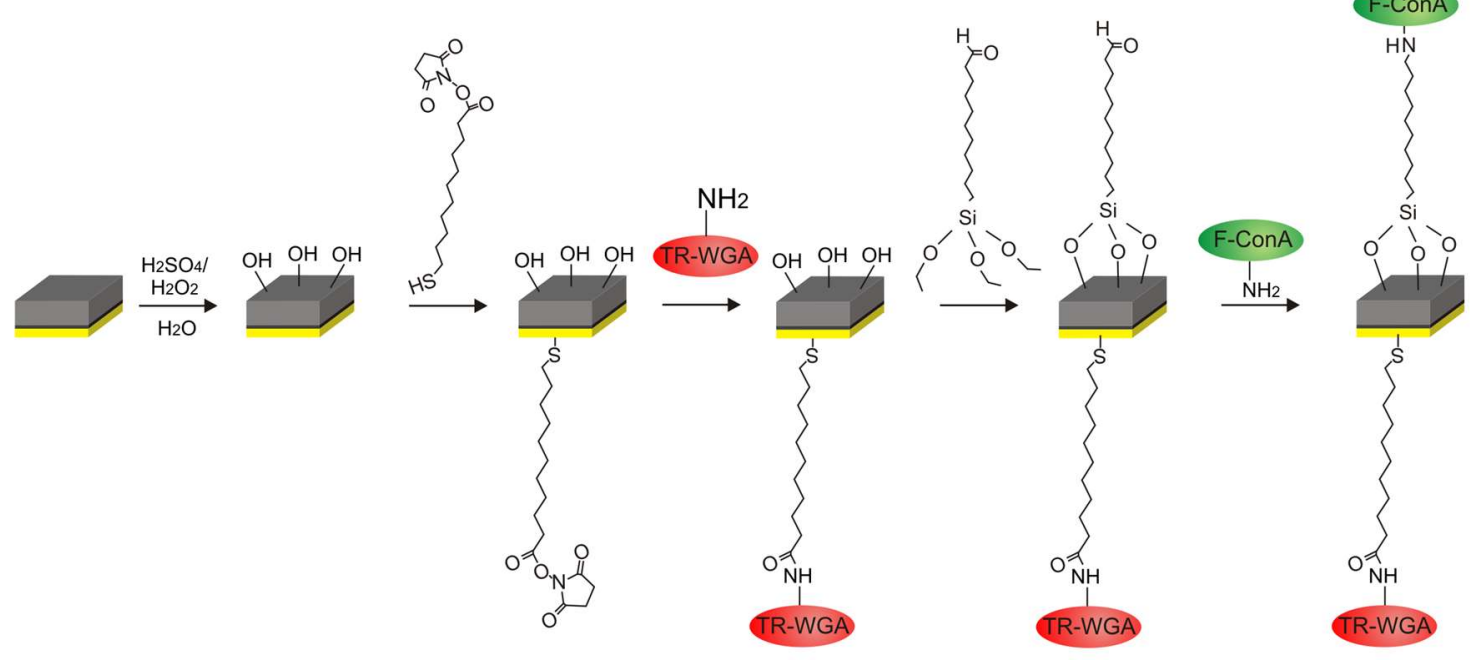

Figure 6. Stepwise formation of double biofunctionalized chips. a) Polysilicon-chromium-gold chip, b) Polysilicon activation, b) mercaptoundecanoate-NHS SAM formation on gold, c) TRWGA immobilization d) TESUD SAM formation on polysilicon and e) F-ConA immobilization. 

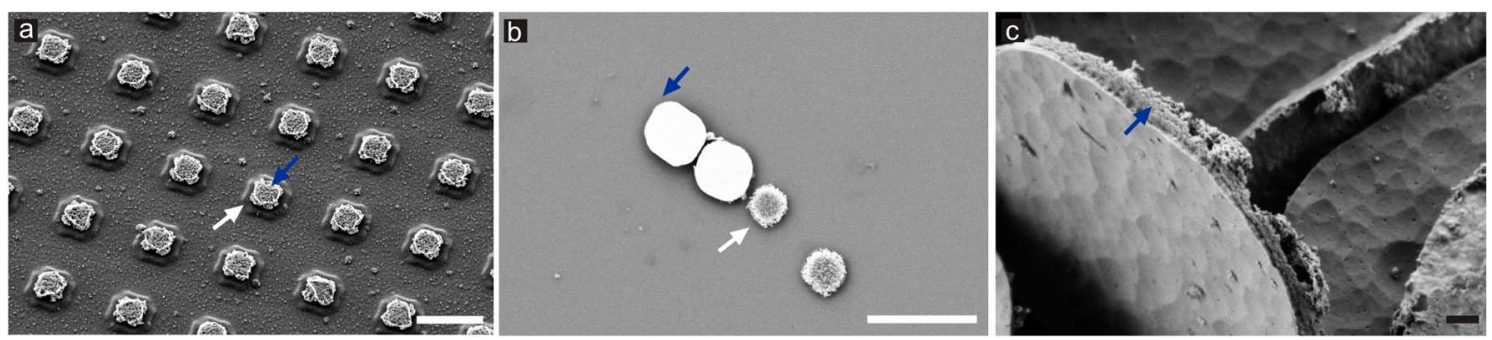

Figure 7. Fabrication results of polysilicon-gold chips. a) SEM image of the chips still adhered on the wafer. A ruined polysilicon patterning was obtained due to the non-defined edges of the gold layer caused by the bad adherence between polysilicon and gold layers. As this material should act as a mask during the polysilicon dry etching and homogenous gold edges were necessary to define the devices. b) Polysilicon-gold chips after their wafer release. Gold and silicon layers were completely separated. c) A released chip zoom image of the ruined edges of the gold layer. White arrows point out silicon. Blue arrows point out gold. White scale bar $=5$ $\mu \mathrm{m}$. Black scale bar $=200 \mathrm{~nm}$. 

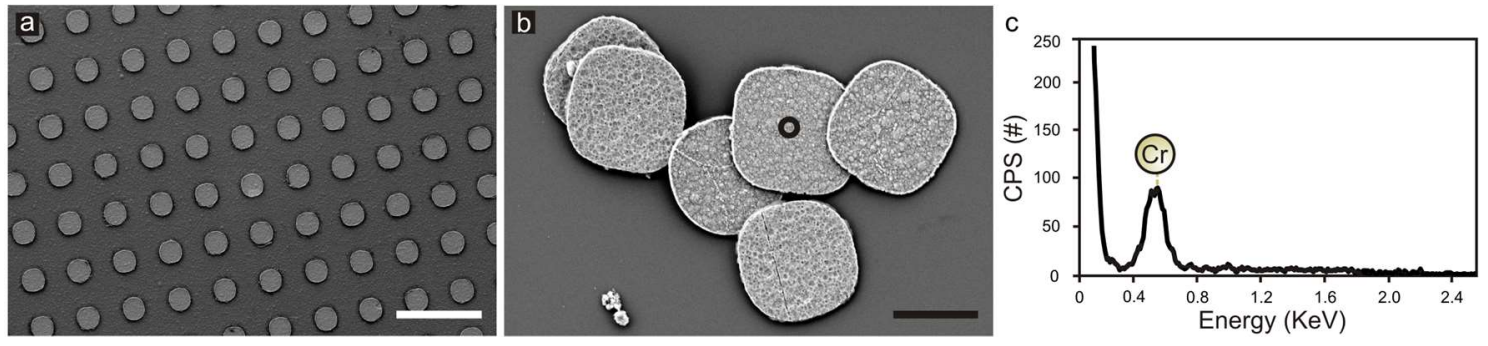

Figure 8. Fabrication results of chromium chips. a) SEM image of the wafer before and b) after chip release. Black encircled area points out where the EDX analysis was performed. c) EDX spectrum confirmed chromium presence. Chromium X-Ray signal can be observed at 0.573 $\mathrm{KeV}$. White scale bar $=10 \mu \mathrm{m}$. Black scale bar $=2 \mu \mathrm{m}$. 

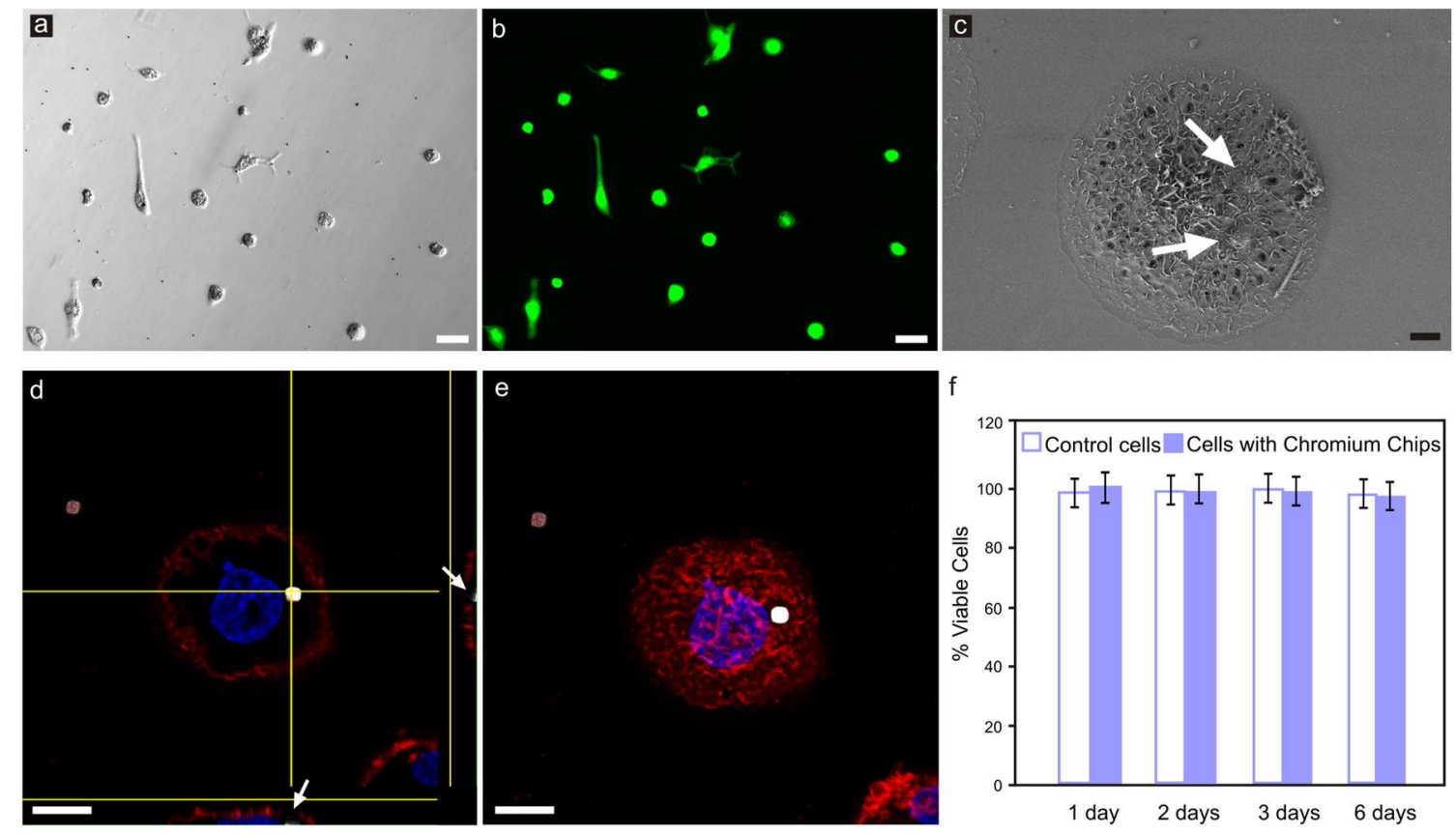

Figure 9. Cell-viability assays with internalized chromium chips. a) Inverted microscope images of macrophages incubated with chromium chips, nearly all the cells are in contact with a chip. Scale bar $=50 \mu \mathrm{m} . \mathrm{b}$ ) The same image under the inverted fluorescence microscope shows that all cells are alive (green cells) and no dead cells (red cells) are observed, using calcein acetomethoxy and ethidium homodimer-1, respectively. Scale bar $=50 \mu \mathrm{m}$. c) SEM image of a cell with two internalized chromium chips. Arrows indicate zones where intracellular chips are situated. Scale bar $=5 \mu \mathrm{m}$. d) Orthogonal projection of the z-stack reconstructions of consecutive focal planes showing a culture cell with an internalized chip. Chips appear as white spots in confocal laser images due to the higher reflectivity of the polysilicon. White arrows indicate the internalized chip. Scale bar $=10 \mu \mathrm{m}$. e) Maximal confocal projection of the same cell taken with CLSM. Scale $\mathrm{bar}=10 \mu \mathrm{m}$. f) Percentage of viable cells in the presence or absence (control) of chromium chips (Mean \pm Standard error of the mean), $n=750$. 

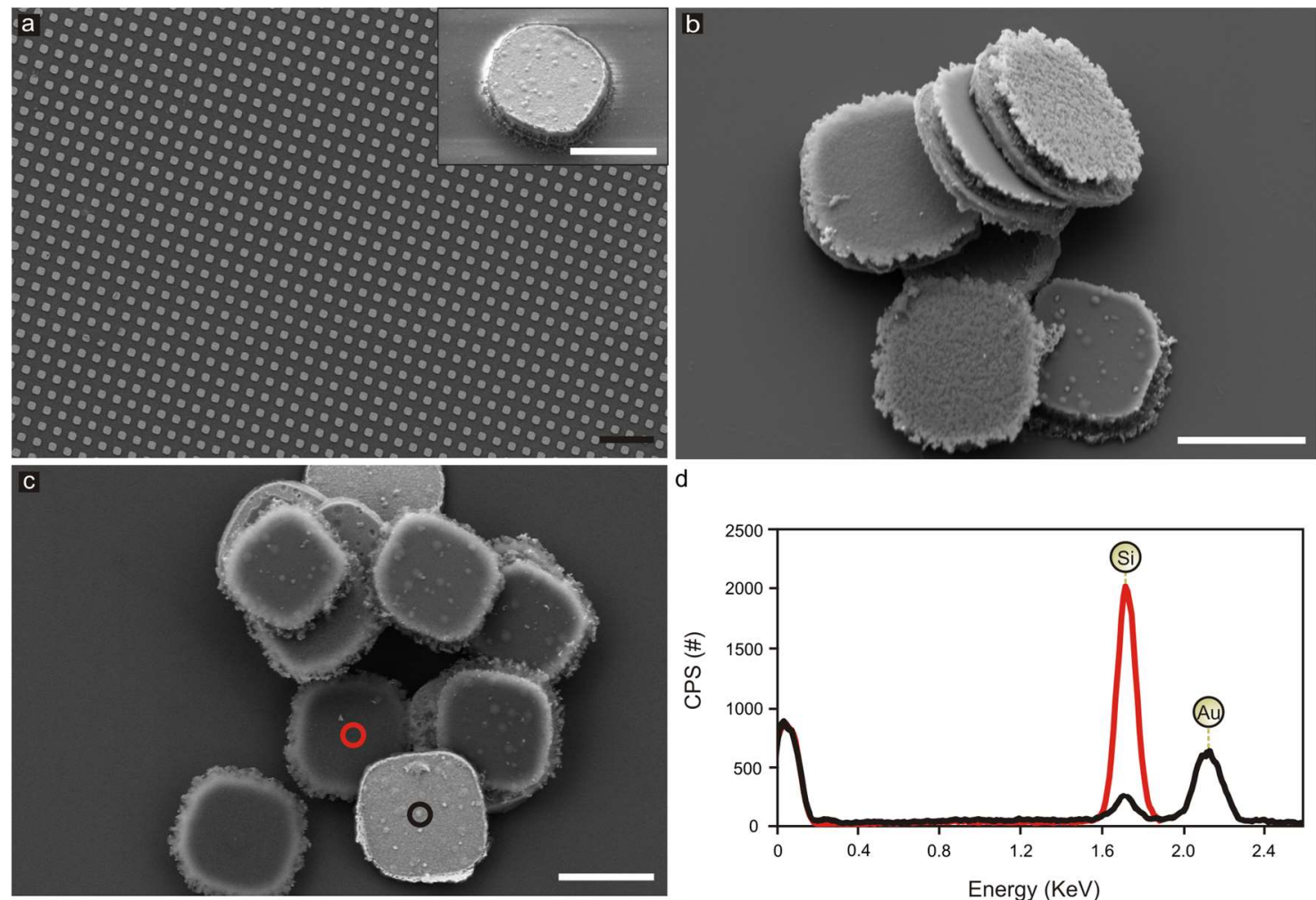

d
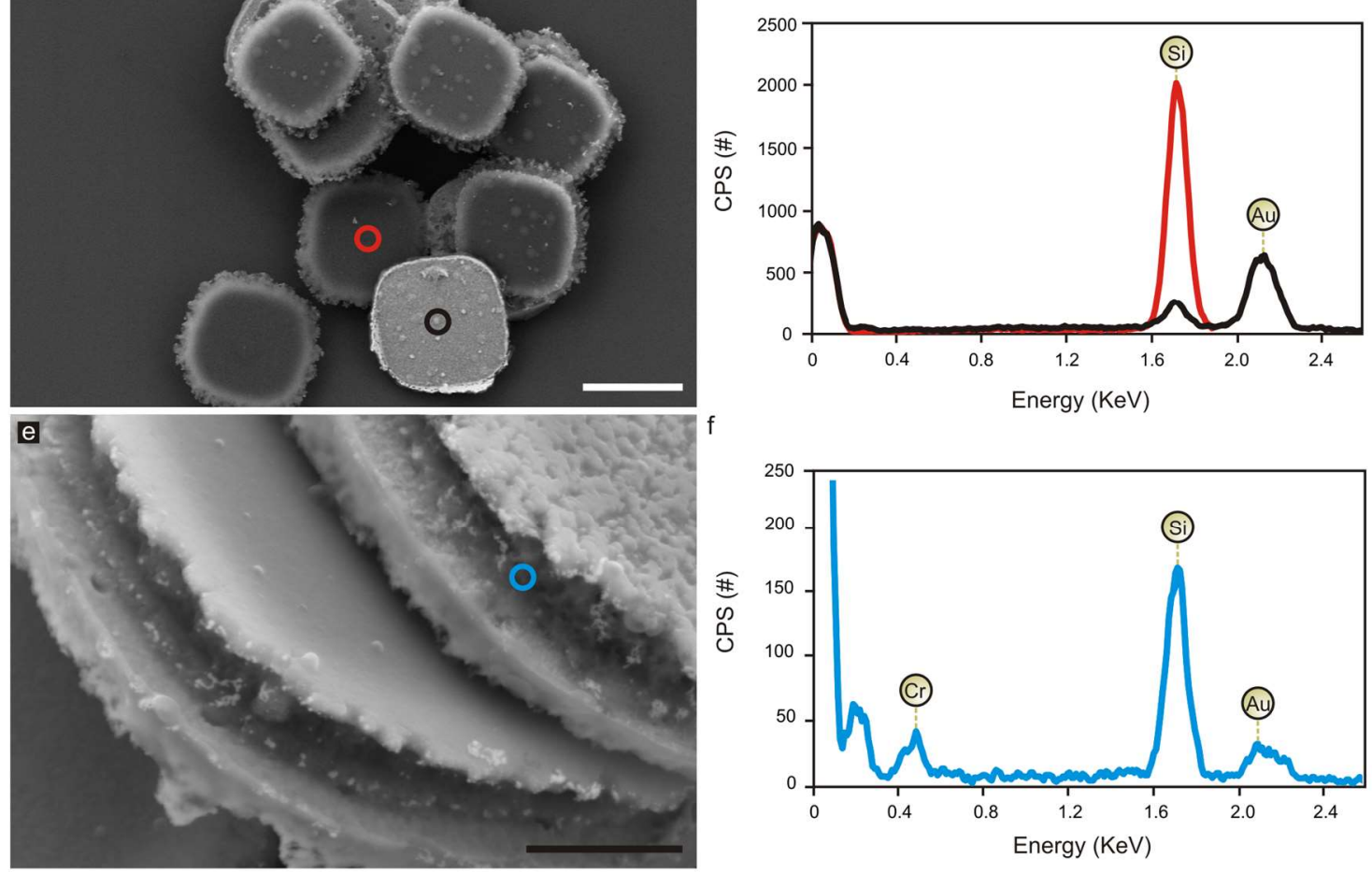

Figure 10. Fabrication results of polysilicon-chromium-gold chips. a) SEM image of the wafer before (inset is a zoom image) (White scale bar $=2 \mu \mathrm{m}$. Black scale bar $=20 \mu \mathrm{m}$ ) and b) after polyslicon-chromium-gold devices release. Scale bar $=2 \mu \mathrm{m}$. c) Released multi-material chips (black and red encircled areas indicate gold and polysilicon layers, respectively and point out where the EDX analysis were performed). Scale bar $=2 \mu \mathrm{m}$. d) EDX spectrum confirmed polysilicon and gold presence. e) Tilted released multi-material chips (blue encircled area indicates chromium layer and where the EDX analysis was performed. Scale bar $=500 \mathrm{~nm} . \mathrm{f}$ ) EDX spectrum confirmed polysilicon, chromium and gold presence. Chromium, polysilicon and gold EDX signals can be observed at $0.573 \mathrm{KeV}, 1.739 \mathrm{KeV}$ and $2.120 \mathrm{KeV}$, respectively. 

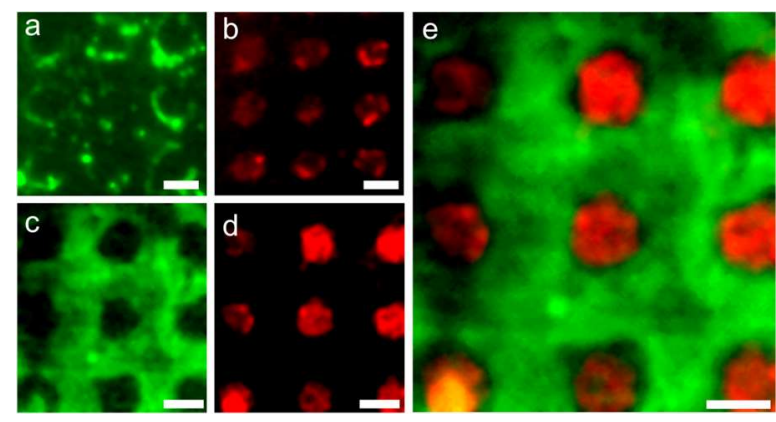

Figure 11. Fluorescence microscopy images of polysilicon-chromium-gold wafer orthogonally functionalized with F-ConA (green) and TR-WGA (red). a) Green fluorescence indicates a FConA mono-functionalized polysilicon surface. b) Red fluorescence indicates a TR-WGA monofunctionalized gold surface. c) Bi-functionalized surface showing F-ConA green fluorescence. d) Bi-functionalized surface showing TR-WGA red . e) Overlap of $\mathrm{c}$ and d images. Scale bar $=3$ $\mu \mathrm{m}$. 

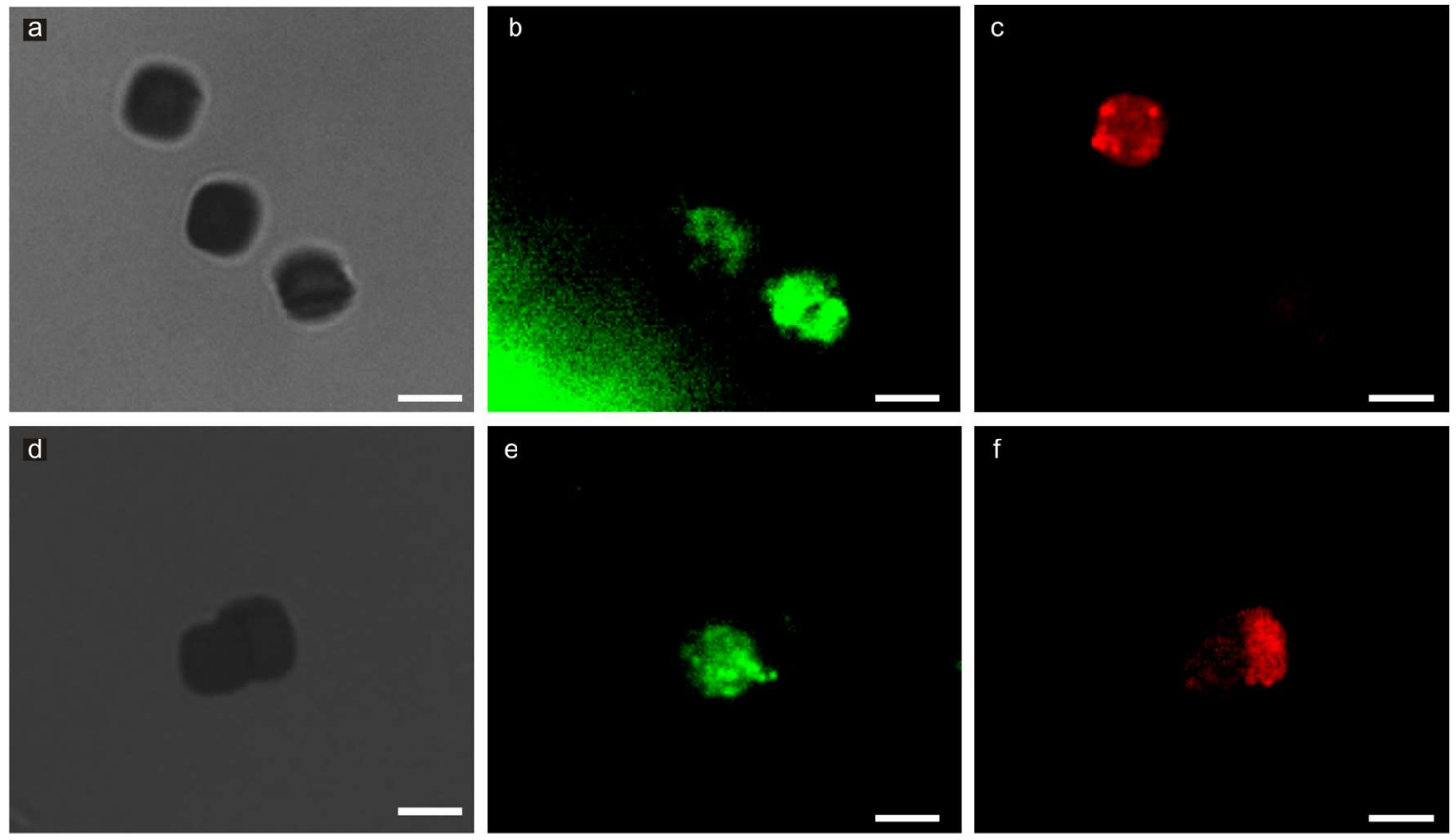

Figure 12. Fluorescence microscopy images of released polysilicon-chromium-gold chips orthogonally functionalized with F-ConA (green) and TR-WGA (red). Microscopy images of several chips: (a-c) several isolated chips and its corresponding a) optical and b) fluorescence microscopy images obtained in green and c) red emission; (d-f) overlapping chips and its respective d) optical, e) green and f) red fluorescence images. Scale bar $=3 \mu \mathrm{m}$. 

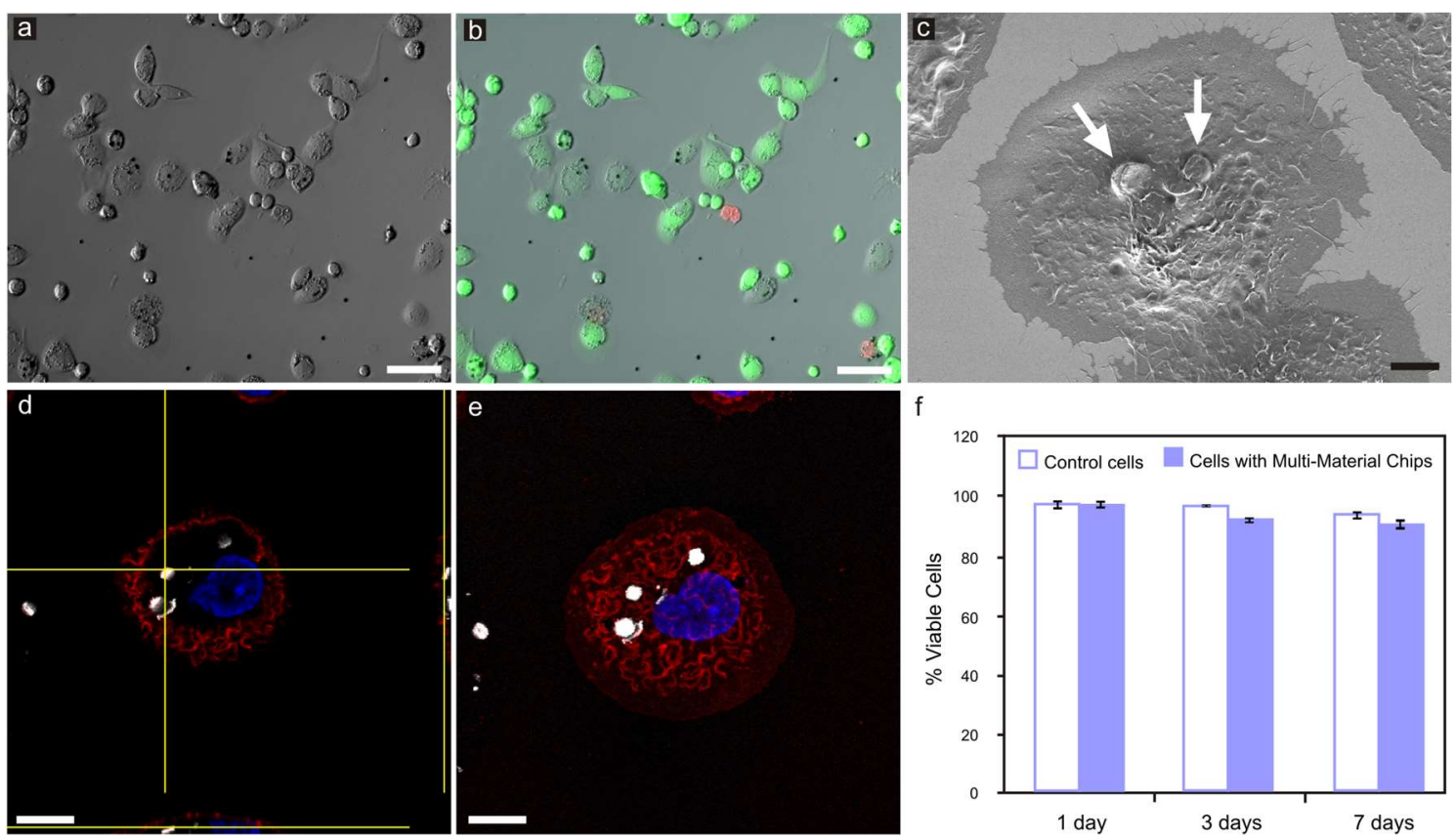

Figure 13. Cell-viability assays with internalized polysilicon-chromium-gold chips. a) Inverted microscope images of macrophages incubated with polysilicon-chromium-gold chips. Scale bar $=50 \mu \mathrm{m} . \mathrm{b})$ The same image observed under an Inverted fluorescence microscope shows that most of the cells are live (green fluorescence) while scare ones are death (red fluorescence). Scale bar $=50 \mu \mathrm{m} . \mathrm{c})$ SEM image of a cell with several internalized polysilicon-chromium-gold chips. Arrows indicate zones, where intracellular chips are situated. Scale bar $=5 \mu \mathrm{m}$. d) Orthogonal projection of the z-stack reconstructions of consecutive focal planes with several internalized chips. Chips appear as white spots in confocal laser images. Scale bar $=10 \mu \mathrm{m}$. e) Maximal confocal projection of the same cell taken with CLSM. Scale bar $=10 \mu \mathrm{m}$. f) Percentage of viable cells in the presence or absence (control) of multi-material chips (Mean \pm Standard error of the mean), $n=750$. 

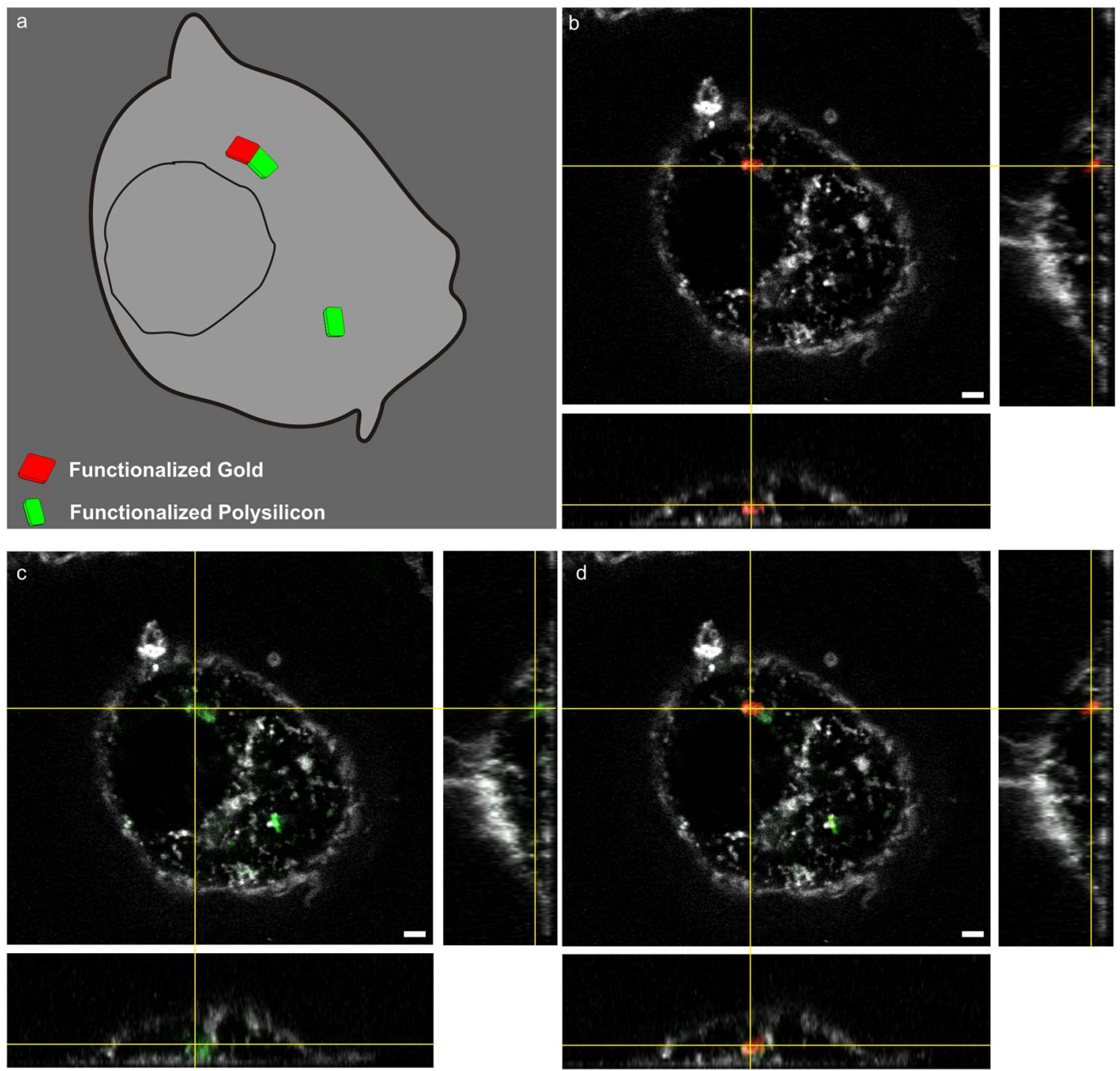

Figure 14. Cell internalization of bi-functionalized polysilicon-chromium-gold chips. a) Conceptual representation of functional polysilicon-chromium-gold chips inside a living macrophage. b-c) Orthogonal projections of the z-stack reconstructions of consecutive focal planes with three internalized chips. Devices appear as red and green spots in confocal laser images, depending on which layer, gold or polysilicon, were exposed. Cell membrane is shown in grey. d) Merged channels of both fluorescence signals. Oregon green was immobilized on the gold using cysteamine and Bodipy 581/591 was immobilized on polysilicon by the aminoundecyltriethoxysilane Scale bars $=5 \mu \mathrm{m}$. 